\title{
Opposition und SED in der Friedlichen Revolution: wertvoller Erkenntnisgewinn in doppelter Hinsicht
}

\author{
Gutzeit, Martin, Helge Heidemeyer und Bettina Tüffers (Hrsg.): Opposition und SED in der \\ Friedlichen Revolution. Organisationsgeschichte der alten und neuen politischen Gruppen \\ 1989/90, Verlag Droste, Düsseldorf 2011, 263 Seiten, 29,80€.
}

Neue parlamentarische Systeme entstehen zumeist nach tiefgreifenden Umbrüchen oder im Gefolge kriegerischer Auseinandersetzungen. Dann öffnen sich Gelegenheitsfenster, die es erlauben, in einem kurzen historischen Moment jenseits von Pfadabhängigkeiten gänzlich neue Ordnungen zu etablieren. Die friedliche Revolution in der DDR im Herbst 1989 hat einen solchen Moment geschaffen. Diese Möglichkeitsräume der Politik hat Joachim Gauck im Rückblick als „einen Traum vom Leben“ beschrieben, der „ganz unerwartet wirklich“ wurde. ${ }^{1}$ In ihrem unblutigen Verlauf gab die Dynamik der Revolution dem Experimentellen der Politik seinen Raum. Dabei handelte es sich um eine geschichtliche Periode, in der die Oppositionellen als gesellschaftliche Pionierakteure - begleitet von einem gewissen „Hoffnungsüberschuss“ (S. 229) - auf den Trümmern der alten Ordnung handelten.

Der vorliegende Band dokumentiert die Tagung „Opposition und SED in der Friedlichen Revolution. Organisationsgeschichte der alten und neuen politischen Gruppen und Parteien", die im November 2008 in Berlin stattfand. Ziel der Konferenz war es, den Ertrag der politik- und zeitgeschichtlichen Forschung der vergangenen zwanzig Jahre sichtbar zu machen und ihn durch Diskussionen mit den damaligen Akteuren zu beleuchten.

Gegliedert in sieben thematische Abschnitte beleuchtet der Tagungsband die Parteineugründungen im Gefolge der Revolution vom Herbst 1989, die Reaktion der herrschenden SED, die Arbeit des Zentralen Runden Tisches sowie die Volkskammerwahl vom 18. März 1990 und ihren Beitrag zur Herstellung der deutschen Einheit.

Die friedliche Revolution in der DDR gehört zu den Sternstunden der jüngeren deutschen Demokratiegeschichte. Getragen wurde dieser epochale Umbruch durch die unterdrückten Bewohner der DDR, die plötzlich zu Bürgern wurden - Bürger, die es wagten, offen ihre Opposition zum SED-Regime zu artikulieren, überfällige Reformen zu erzwingen und sich damit den Weg zu Freiheit und Demokratie erkämpften. War die erste Phase der Revolution durch das Begehren nach Freiheit, Demokratie und partizipatorischem Einfluss gekennzeichnet, ging es bereits nach wenigen Wochen darum, der Protestbewegung eine organisatorische Form zu verleihen. Rasch erwuchsen aus den Oppositionsgruppen politische Parteien, die bislang nicht artikulierte Interessen bündelten, Personal rekrutierten und eine schier unüberschaubare Fülle an plötzlich auftretenden Fragen zu Parteiprogrammen verdichteten. Auf der Unterseite des unblutigen Umsturzes entstand eine revolutionäre Großzügigkeit, indem die alte Nomenklatura nicht brutal liquidiert, sondern - in Anzahl und Einfluss geschwächt - durch die neuen institutionellen Arrangements des Parlamentarismus eingehegt wurde. Zwar war die Einparteienherrschaft der SED überwunden, doch blieb ihr die Chance nach inhaltlichen und strukturellen Metamorphosen, bei der Wahl zur Volkskammer anzutreten.

1 Rede von Joachim Gauck, Bundesbeauftragter für die Unterlagen des Staatsicherheitsdienstes der ehemaligen DDR, anlässlich der Sonderveranstaltung „10. Jahrestag des Mauerfalls“ im Deutschen Bundestag am 9. November 1999. 
Als ausgewiesener Kenner der Revolutionsgeschichte der Jahreswende 1989/90 beschreibt Ilko-Sascha Kowalczuk die Lage in der DDR am Vorabend des revolutionären Umbruchs. Eine im Kern erstarrte Gesellschaft, ein hypertrophes und im Niedergang begriffenes Wirtschaftssystem waren die sichtbarsten Zeichen einer zugespitzten Krisenlage, die alle Gesellschaftsbereiche erfasst hatte. Mit der großen Demonstration am 9. Oktober 1989 in Leipzig begann die Zeit der Friedensgebete, Montagsdemonstrationen und Protestveranstaltungen. Damit konstituierte sich erstmalig in der DDR, wie der Historiker Karsten Timmer feststellt, eine neugewonnene „Öffentlichkeit massenhaft als herrschaftsfreier Diskurs“ (S. 91).

Als Ergebnis der gelungenen Revolution, die die SED in ihrem Alleinvertretungsanspruch delegitimiert hatte, stand als institutioneller Ausdruck die Einberufung des Zentralen Runden Tisches. Mit dem Fall der Berliner Mauer und der machtpolitischen Schwächung der SED war die staatliche Gesamtarchitektur der DDR deutlich unterspült worden. In dieser Situation garantierte der Runde Tisch die Einbindung der Bürgerrechtsorganisationen in die kollektive Verantwortung für die aktive Gestaltung des Umbruchsprozesses. Nach polnischem und ungarischem Vorbild organisiert war der Runde Tisch zunächst „ein ideelles Importprodukt" (S. 148) und sollte eine Verfassungsreform und in einem zweiten Schritt freie Wahlen in der DDR vorbereiten. Am Horizont der sich immer wieder neu beschleunigenden Ereignisse konkretisierte sich bald die Perspektive der Einheit und in diesem Sinne war, wie Martin Gutzeit als damaliger Vertreter der SDP heute resümiert, der Runde Tisch „eine Übergangsinstitution mit Verfallsdatum“ (S. 160).

Aus dem Geist der erfolgreichen Revolution ist mit den ersten freien Wahlen zur Volkskammer am 18. März 1990 ein neuer Parlamentarismus auf dem Boden der DDR entstanden. Diese Entwicklung, die die drei Herausgeber im Vorwort einen „Prozess der ,Selbstparlamentarisierung“" nennen, gehört zu den zentralen Marken auf dem Weg zur staatlichen Einigung im Oktober 1990. Während im Deutschen Bundestag ein hoher Grad an Professionalisierung durch den Typus des Berufspolitikers vorherrscht, „rekrutierte sich die Volkskammer vor allem aus einer breiten sozialen Elite von Ingenieuren, Naturwissenschaftlern, Ärzten, Lehrern, Geisteswissenschaftlern und Pfarrern" (S. 16). Die Arbeit der Volkskammer spiegelte allzu deutlich, wie sich revolutionäres Geschehen in parlamentarische Verfahren und konkrete Abstimmungsergebnisse übersetzen ließ. Aus westdeutscher Sicht wurde das frei gewählte und reformierte DDR-Parlament zwar als Truppe von „Laienspielern“ verspottet, doch aus der unmittelbaren Erfahrung der Akteure von damals als „Laboratorium der Politik"2 gelobt.

Gunnar Peters wirft einen Blick auf die Arbeitsweise der Volkskammer, die er als „ein Kurzparlament der deutschen Geschichte“ (S. 199) bezeichnet. Auffallend ist, wie sehr Sachbezogenheit und die Kraft des besseren Arguments über parteipolitisch motivierte Auseinandersetzungen triumphierten. Die Leistung der Volkskammer lag nicht zuletzt in einer kollektiven Lernerfahrung unter dem Eindruck ,unzulänglicher parlamentarischer Infrastruktur in Ost-Berlin“ (S. 208). „Was die Häufigkeit und Ausprägung von Zwischenrufen angeht“, so beobachtet Peters, „ähnelte die letzte Volkskammer weniger dem Bundestag, sondern mehr der Frankfurter Paulskirche 1848/49“ (S. 205). Seine Gesamtbeurteilung dieses Parlaments fällt insgesamt sehr positiv aus: „Das Theaterstück, das die Laienspieler auf einer asbestbelasteten Kleinkunstbühne in Ost-Berlin gaben, unterschied sich nicht wesent- 
lich von dem der Profis im traditionsreichen Bonner Staatsschauspiel“ (S. 208/209). In der anschließenden Podiumsdiskussion der Tagung blickt Werner Schulz, damals Parlamentarier des Neuen Forums, kritisch auf die politische Arbeitsleistung der Volkskammer zurück. In der Rückschau beklagt er, dass die situative Dynamik nicht ausreichend in Politik umgesetzt worden sei. Die Ambitionen der Volkskammer waren gering, „weil hier mehr oder weniger der kollektive Ausreiseantrag betrieben wurde" (S. 226).

Die abschließende Diskussion verdeutlicht die Bedeutung der friedlichen Revolution des Herbstes 1989 für die Politische Kultur des vereinigten Deutschlands. Die Akteure der Revolution hatten die Gunst des geöffneten Fensters der Geschichte erkannt. Sie hatten gelernt, so der Bonner Staatsrechtler Josef Isensee, „dass man die günstige Gelegenheit, um die staatliche Einheit zu erlangen, rasch ergreifen muss und nicht, wie vormals in gelehrten Diskursen über die bestmögliche Verfassung, die einmalige Chance zur Einheit verstreichen lassen darf, so dass der Verfassungsentwurf zu Archivmaterial gerät“ (S. 236).

Die turbulenten Revolutionsmonate zwischen dem Fall der Mauer und der Volkskammerwahl glichen, wie der Historiker Bernd Florath urteilt, einer „verdichteten Zeit“ (S. 130). Die Vorträge, Diskussionen und Redebeiträge der Tagung machen diese Ereignisdichte noch einmal plastisch und unmittelbar begreifbar.

Für die Parlamentarismusforschung liegt mit diesem Band ein wertvoller Erkenntnisgewinn in doppelter Hinsicht vor: Zum einen wird deutlich, wie parlamentarische Neugründungen in Transformationsprozessen eines nicht-demokratischen Systems gelingen können. Zum anderen wird durch die Diskussionen am Ende eines jeden Podiums sichtbar, welche Gestaltungsoffenheit revolutionären Momenten innewohnt und wie ihre oppositionellen Hauptakteure bereit sind, diese Chancen zu nutzen.

Helge F. Jani 